\title{
Childhood Gastric Teratoma: A Case Report
}

\author{
Pankaj Halder*, Kartik Chandra Mandal, Anjan Das and Bidyut Debnath \\ Department of Paediatric Surgery, Post Graduate Institute of Pediatric Sciences, India
}

*Corresponding author: Pankaj Halder (MS, M.Ch), Assistant Professor, Department of Paediatric Surgery Dr. B.C. Roy, Post Graduate Institute of Pediatric Sciences (PGIPS), Saroda Palli, Panchanon Tala, Baruipur, Kolkata-700144, India

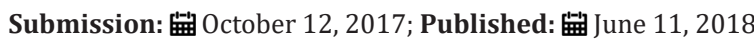

\begin{abstract}
Gastric teratoma is relatively rare type of extra gonadal germ cell tumor which comprises $<1 \%$ of all teratomas. It usually presents with abdominal mass and features of gastric outlet obstruction with or without gastrointestinal bleeding. Complete excision of the tumor is the best mode of treatment. Here, we report a case of gastric teratoma in a two month old male child where we had to go for partial gastrectomy owing to complete removal of the tumor.
\end{abstract}

Keywords: Congenital; Gastric; Teratoma; Melena

Abbreviations: GT: Gastric Teratoma; USG: Ultrasonography; UGI: Upper Gastrointestinal; CECT: Contrast Enhanced Computed Tomography; AFP: Alpha-Fetoprotein; SOL: Space Occupying Lesion

\section{Introduction}

Teratoma, a true neoplasmarises from totipotential cell and occurs in the ovary and testis. Extra-gonadal teratoma occurs in descending order of frequency in sacrococcygeal region, mediastinum, intracranial region \& retroperitoneum respectively. Gastric teratoma (GT) is one of the rarest site for extra gonadal teratoma. Apart from clinical findings, ultrasonography (USG), upper gastrointestinal (UGI) contrast study and contrast enhanced computed tomography (CECT) do play important role in the diagnosis of gastric teratoma. However, diagnostic utility of alpha-fetoprotein (AFP) is less in young children because of normal physiological elevation [1]. GT contains both cystic and solid components with or without calcifications. As more than $90 \%$ gastric teratomas are benign, no adjuvant chemotherapy or radiotherapy is needed once the tumor is completely excised.

\section{Case History}

A 2 month old boy presented with gradually increasing abdominal mass since birth. He also had a history of nonbilious vomiting and passing black stool (melena) for 7 days. The baby was born to a primi mother by normal vaginal delivery at term. He was $3.6 \mathrm{~kg}$ at birth. Abdominal examination revealed a nontender, mobile, firm to hard mass in the left hypochondrium and epigastrium extending up to the left iliac region across the midline. There was no evidence as cites and the bowel sounds were deviated to the left site of the abdomen. Blood hemogram, serum AFP and liver function test were within normal limit. USG showed a large multi septate cystic space occupying lesion (SOL) in the abdomen. CECT suggested a multi cystic mass $(145 \mathrm{~mm} \times 105 \mathrm{~mm} \times 93 \mathrm{~mm})$ with calcifications in the abdomen. The stomach was shifted to the right and small gut loops were shifted inferiorly and to the left (Figure 1 ) by this heterogeneous mass. In accordance with the reports, we inferred it may be a case of GT.

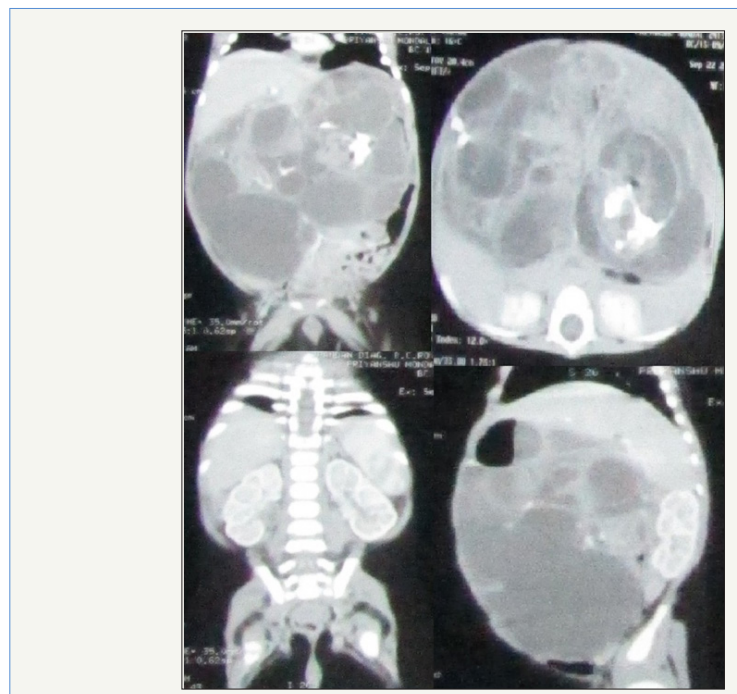

Figure 1: CECT showed a large multi septate cystic lesion with calcifications in the wall. Stomach is shifted to the right, small gut loops shifted inferiorly and to the left.

On exploration, we found a bosselated multicystic lesion arising from the posterior wall of greater curvature of the stomach. It was sitting on the transverse colon by pushing the stomach up 
and duodenum laterally. Omentum was intimately adherent to the tumor. We could separate the tumor from the duodenum and its colonic attachments except a portion of greater curvature. Actually, the tumor mass was originating from the greater curvature (Figure 2). Finally, the mass was fully excised along with $5 \mathrm{~cm}$ gastric wall (site of origin). The stomach was repaired in two layers. Postoperative period was uneventful. We started oral feeding on 5th post-operative day. A repeat USG and AFP after 6 month were normal. There was no evidence of recurrence and patient was doing well in 2 years follow-up. Microscopically, the cyst wall of the tumor was lined by stratified squamous epithelium, goblet cells, ciliated columnar epithelium along with focal calcification. There was presence of mature elements of Skin, sebaceous glands, bone, ganglion cells, cartilage, fat, nerve bundles and hair follicles in the tumor in addition to small and large intestine mucosa which confirmed the diagnosis of mature cystic teratoma.

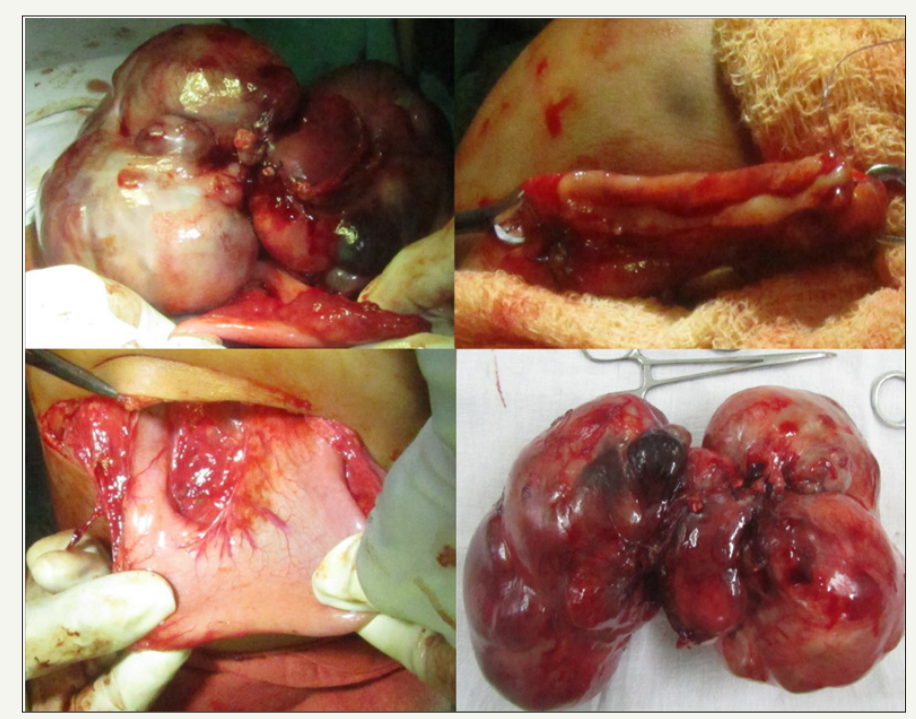

Figure 2: Intra-operative picture showed a huge bosselated mass (both cystic and solid) in the transverse colon pushing the stomach and duodenum. (Before excision, excision with partial gastric resection and excised specimen).

\section{Discussion}

The term teratoma was derived from the Greek word teraton meaning "a monster". It was first described by Virchow in 1869 for a tumor in the sacrococcygeal region. Other sites of extragonadal teratoma are liver, kidney, vagina, stomach, mediastinum and retroperitoneum. The first case of GT was reported in 1922 by Eustermann and Sentry [2]. GT has been hypothesized to originate from pluripotent cells in the gastric wall and contains all three germ cell layers: ectoderm, endoderm, and mesoderm. Though, monodermal type is also described in modern classification. According to recent theories, GT may also arise from extra embryonic cells other than embryonic stem cells, conjoined and mal-developed twins, and undetermined cell types [3-5].

Teratomas are classified as mature or immature on the basis of the presence of neuroectodermal elements within the tumor. Most of the GTs are benign which contain only mature elements as we seen in our case. Sometimes, it contains or develops foci of malignancy. Actually, malignancy at birth is uncommon, but chances of malignant transformation increase with age and incomplete resection of the tumor [6]. The most common malignant component is yolk sac tumor. Clinical differentiation of GT from retroperitoneal teratoma, neuroblastoma, nephroblastoma and pseudocyst of pancreas is difficult because of (i) similar presenting symptoms (ii) Comparable calcification in X-ray or CT. (iii) Corresponding filling defect in the stomach in upper GI contrast study [7]. However, treatment of choice is complete surgical resection of the tumor whenever feasible. Sometimes, a partial gastrectomy is required for removal of entire tumor, as we did in our case. Recurrence of tumor is not described after complete resection. Thus, adjuvant chemotherapy or radiotherapy therapy is not recommended [8]. A grading for teratoma has been described by "Norris grading system". Grade 0- Mature teratoma. Grade 1-presence of immature neuroectodermal tissue in one field of a slide. Grade 2- immature tissue is present in more than one but less than four fields in a slide. Grade 3-immature neuroectodermal tissue is seen more than four fields in a slide. This grading system is not useful in children because the management strategy is not altered by the grade of the tumor [9].

\section{References}

1. Kim SH, Cho YH, Kim HY, Lee YJ, Park JH (2015) Mature Cystic Gastric Teratoma in an Infant: A Case Presenting with a Gastrointestinal Bleeding. J Korean Assoc Pediatr Surg 21(2): 42-45.

2. Ivascu M, Bembea M, Jurca C, Moldovan C, Buicu F (2014) Rare Case of Immature Gastric Teratoma. Acta Medica Marisiensis 60(4): 167-170.

3. Gupta DK, Srinivas M, Dave S, Agarwala S, Bajpai M, et al. (2000) Gastric teratoma in children. Padiatr Surg Int 16: 329-332.

4. Al-Salem AH (2012) Immature gastric teratoma in a newborn. Med Case Stud 3: 12-16.

5. Herman TE, Siegel MJ (2008) Congenital gastric teratoma. Journal of Perinatology 28: 786-787. 
6. Corapcioglu F, Ekingen G, Sarper N, Guvenc BH (2004) Immature Gastric Teratoma of Childhood: A Case Report and Review of the Literature. J Pediatr Gastroenterol Nutr 39: 292-294.

7. Ramos MCV, Méndez ALM, Contreras CAR, Montes CER (2010) Pediatric Gastric Teratoma. Radiology Case 4: 6-13.
8. Kishore P, Jain P (2015) Malignant gastric teratoma in a 3-month-old infant. Indian J Med Paediatr Oncol 36(4): 271.

9. Deodhar KK, Suryawanshi P, Shah M, Rekhi B, Chinoy RF (2011) Immature teratoma of the ovary: A clinic pathological study of 28 cases. Indian J Pathol Microbiol 54(4): 730-735.

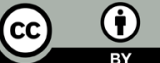

Creative Commons Attribution 4.0 International License

For possible submissions Click Here

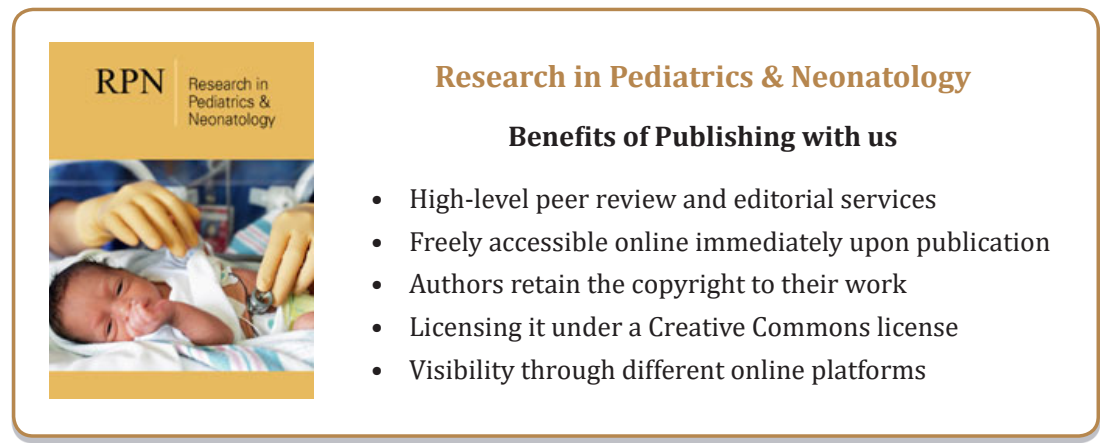

